\title{
20
}

\section{Verification of Large Systems in Silicon}

\author{
Cary Ussery and Simon Curry \\ Cadence Design Systems Inc. \\ 270 Billerica Road \\ Chelmsford MA 01824. USA
}

Phone: (508) 446-6308 Fax: (508) 446-6665

Email: cary@cadence.com, curry@cadence.com

\begin{abstract}
Two new trends are emerging in the design and production of very large integrated systems on silicon: on the one hand technology that enables a new opportunity for trade in encapsulated intellectual property cast into re-usable blocks for system-level use and, on the other, the partition of the vertical flow from high-level design through to manufacture into at least three separate businesses - the system design house, the IP provider and the silicon implementation house. While the situation appears to be similar to that which prevailed at lower scales of integration (e.g. TTL and boards), the complexity and scale of the new systems and their components pose interesting challenges for designers and the businesses in which they operate. This paper considers, in particular, the challenges that relate to design and verification flow and some possible consequences for the evolution and use of HDLs.
\end{abstract}

\section{Keywords}

System-Level Specification, System Level Design, Verification, Validation, Hardware Description Languages

\section{INTRODUCTION}

The design of electronic systems is undergoing a fundamental change. This is underscored by two significant and related trends: the ability to produce complete 
systems and subsystems on a single chip and the growing availability of encapsulated intellectual property (IP) which can be used to construct these systems. These trends promise enormous productivity, efficiency and economic gains as the builders of highly integrated systems take advantage of them.

These technology trends are also driving new business dynamics into the electronics design market. In particular, the relationship between system design houses and silicon providers will undergo major modifications; and we are witnessing the emergence of IP providers who may or may not be in the business of producing their own chips.

It is clear that as increasing amounts of design content come in the form of encapsulated intellectual property, the real systems design challenge is to effectively evaluate and integrate IP blocks and to verify them in the context of the overall system. This design then needs to be packaged and transferred to a silicon implementation factory.

While business trends are pressing for a clean interface and hand-over between IP authoring and implementation on the one hand, and system-level design and integration on the other, the impact of semiconductor process technology trends, towards $0.35 \mu$ and below, is making the crispness of the interface much harder to manage since it is becoming increasingly necessary to be aware of physical effects even when performing high-level design (RTL \& above).

In attempting to assess the directions for development of HDLs, it will be helpful to analyze their roles in evolving design flows. We identify two broad classes of flow that we label 'HDL-Based' and 'Block-Based' design flows.

\section{HDL-Based Design Flows}

The evolution of these flows seems to be from a waterfall approach (RTL, Logic, Layout) situation to one of increasingly abstract behavioral descriptions accompanied by synthesis technology that is tuned to the broader range of descriptions.

Success depends on productivity gains that arise from behavioral synthesis, HDLbased libraries and code re-use, and enhanced back-end flows that permit a better concurrent-engineering approach for partitioning, synthesis and floorplanning in order to control the increasing influence of interconnect on structure and timing.

Attempts are being made to bridge the gap between system-level/architectural design and implementation by generating synthesizable HDL descriptions from higher level representation and by the use of libraries of 'hard-macros' and block descriptions that can drive more specialized circuit generators.

\section{Block-Based Design Flows}

Block-based design flows represent an attempt to respond to a new set of drivers in the marketplace:

- The emergence of huge integrated systems on silicon $\left(10^{6}-10^{7}\right)$ gates. 
- Pressure to shrink the design interval to months rather than years

- The mixed functionality that these systems require is often not covered by the core-competencies of any single organization.

- Intellectual property (IP) encapsulated in a variety of design delivery standards and the potential for trade in such goods (VSI Alliance, 1996).

- New market participants and dynamics: IP integrators, IP authors and silicon foundries.

Block-based design flows incorporate HDL-based design and verification subflows, but the emphasis of the design and verification effort shifts to the system-level. Requirements on the HDL-based sub-flows make demands that current HDL architectures do not adequately satisfy and render redundant some features of HDLs that have seemed important until now.

A 'New' Flow Paradigm

Until recently, system architects and system implementers lived in different spaces. System architecture was defined and verified using lightweight representations and languages; and then the architecture was communicated, usually in a written document, to the 'design' and implementation community. The impact of deepsubmicron implementation technology, the need for rapid and reliable progress to market, block-based design and imported IP force these two disparate communities into one flow. The 'New' epithet in the title thus recognizes that flows of this type have been in operation, albeit in a broken way, for some time.

We identify four major stages or levels in the flow that are particularly appropriate for Block-based flows but apply as well for the future of HDL-based design: System, Mapping, Architecture, Implementation. These fit well with classifications that others have made, for example Shi (1996). The characteristics of these four stages are:

System - This stage is for checking the validity of the algorithms chosen for the product. Models are typically developed in either block-level graphical environments or in $\mathrm{C}, \mathrm{C}++$ and similar languages.

Mapping - In this stage, the algorithm is mapped into an organization whose elements may have concurrent and overlapping duty cycles and which may be chosen for implementation either as hardware or software for one or more instruction-set processors. Modeling at this level has traditionally been difficult. The most successful approaches have employed discrete-event, queue-oriented systems to analyze the performance characteristics of the system.

Architectural - In this stage, the mapping is completed by a representation that is amenable to direct translation to implementation. HDLs at the behavioral and RTL 
levels will be used for cycle-accurate models which can, in many cases, be synthesized into logic. In a block-based flow we expect heavy use of predefined large blocks and block descriptions that are transformed by special-purpose generators. We observe that in both flow classes and, particularly emerging block-based flows, the HDL that is used comes from libraries or generators from higher levels. Software at this level is typically in $\mathrm{C}$ or $\mathrm{C}++$ although we expect to find increasing use of Java for embedded systems.

Implementation - This stage produces the 'sign-off-ready' representation of the product with which the functional tests, that remain after verification at the preceding levels, may be carried out. In particular, timing-related verification occurs at this level. Shi et al.(1966) make the point that verification models at these levels run progressively more slowly as design passes from system to implementation. In his case:

system: mapping: architecture: sign-off $=1: 100: 18000: 27000$

although the correspondence between his categories and ours is approximate.

\section{Expected future use of HDL-Based and Block-Based Flows}

We believe that HDL-based flows will continue to enjoy investment and growth for a few years to come but that they will not be able to rise to the challenge of rapid and reliable delivery of the complex chips that the consumer market will demand. Their use, and the importance of HDL-based design, will therefore diminish in favor of Block-based design approaches, although there will continue to be roles for these flows for small chips ( $<300 \mathrm{~K}$ gates) and for some of the authoring process required by Block-based design.

The rest of this paper aims to justify this assertion by exploring the verification issues and required design representations to enable IP-based design.

\section{DESIGN FLOW AND DESIGN MODEL CLUSTERS}

Figure 1 summarizes the major levels in an overall system design flow. Each design abstraction level represents a major view of the system which allows the designer to define, analyze and verify specific characteristics of the system.

Traditionally these have been viewed as different 'abstraction' levels; however, it is more appropriate to think of them merely as different representations of the system which focus on different characteristics.

Typically, higher levels focus on sophisticated modeling of the environment (or context) surrounding the target system using abstract data representations (e.g., floating point). Lower levels tend toward more physically-oriented views of data and 
the system with constrained modeling of the surrounding environment. This is natural as the design characteristics being explored move from function within an environment to implementation on a specific target architecture.

This paper addresses the design and verification from the system level to implementation; product specification and detailed implementation are outside the scope of the paper. Each level of design abstraction requires different views of a system or design and different verification techniques.

\subsection{Modeling}

Effective verification and validation of a design requires building models of the environment in which the design must operate. With the market demand for sophisticated multi-function, flexible electronic products, systems must handle multiple complex communication protocols, data representations, processing algorithms and interactivity demands. Nowhere is this more apparent than in the design of electronic personal communication systems which must handle audio, video and data transmitted through multiple protocols with real-time decompression, signal processing and user interaction. A design team needs to verify its product in this complex environment and, therefore, must use models of the environment.

Fortunately, the task of modeling is greatly simplified by the availability of predefined libraries of models which can be used to construct environment models, system-level descriptions and hardware and software target architectures. These models come from model and tool suppliers as well as from silicon implementation houses (e.g., ASIC, FPGA and merchant IC suppliers). One of the primary goals of the VSI Alliance (1996) is to define a standard infrastructure in which models of intellectual property can be provided which work effectively with the tools and methodologies used to incorporate IP into an overall electronic system. In addition, many software vendors provide predefined libraries of routines which can be used in the implementation of system software. This is especially common for signal/data processing algorithms and for implementing standard protocols (protocol stacks). With all these types of models, the primary task of modeling is fast becoming a job of composing a system model out of a collection of building blocks.

Given the complexity and number of models required for the tools and methodologies used throughout a system design, it is unreasonable to expect to use a single modeling language or mechanism. The goal of a modeling environment or language should be to allow intuitive capture of a design and the ability to be used effectively and efficiently by the tools required to support the design team's methodology. There is a fair amount of tension between these two goals in any modeling environment. Throughout the paper, we will describe the most common modeling capabilities used at different stages in the design process. 


\subsection{Verification}

Clearly verification is a key, if not the primary, challenge in system design. The complexity of electronic systems and the environments in which they must operate has never been higher. Systems must be designed to conform to multiple standard communication protocols, react to complex physical and systemic stimulus and handle large amounts of sometimes lossy data all implemented on system-critical software and hardware. The resulting system must meet performance, power, cost and reliability requirements and be delivered within significantly reduced market windows.

Achieving high test coverage within the available development period is unrealistic and misguided as an ultimate objective. What is needed is a more thoughtful and effective approach to system verification. The key to such an approach is the development of a robust test plan which adopts test strategies and methodologies for the key aspects of the system. Each objective in the test plan represents situations under which the system must operate and detailed development of the expected responses of the system in those situations. We refer to this methodology as 'scenario-based verification'. The main idea is to generate system tests based on how the circuit is expected to operate under real-world conditions.

In addition, various aspects of the system need to be verified within the context of the different components of the system development process. A key aspect of scenario-based verification is understanding what questions to ask about the system to verify which aspects. In particular, a product has various views which are created as part of its development: figure 1 shows an outline of the major views.

A successful test strategy must incorporate a set of test objectives for each of these views which incorporate a collection of test scenarios under which the system must operate. Typical scenarios under which a handset, for example, must operate include: data exchange in conformance with handset to terminal communication protocol standards (e.g., GSM, IS-95); voice quality decoding of input data signals; voice quality encoding of output data signals; smooth transition through a grid of terminals; successful implementation of voice mail features. There are many others but this is sufficient to give a feel for the idea.

Specific scenarios should be developed which will focus on testing these aspects of the design. These scenarios are embodied as a combination of stimulus generators or test vectors, results analyzers or comparators, and a test bench infrastructure for applying the scenario to the design under test. 


\section{SYSTEM-LEVEL DESIGN: HETEROGENEOUS DOMAINS}

System level descriptions are used to capture the essential functionality, major algorithms and environment in which the system operates.

A system description can be viewed as a combination of: functionality or behavior of the system in response to its environment; a system boundary for interacting with its environment; static and/or dynamic models of the environment in which it operates; and constraints on the implementation of the system

\subsection{Major Design Issues}

Algorithm Design - During algorithm design, teams are usually concerned with the impact of given algorithmic decisions on the capability or quality of the product under design. For instance, they might try to assess the impact of a new compression technique on the audible sound produced by the system or evaluate error rates with changes in a signal-to-noise characteristic of a system. Such analyses require applying real-world (or representative) data to the system and examining the dynamic reception, processing, and response of the system to that data. Algorithms are verified by running sequences of data through them. Typically, design teams are interested in the impact of algorithmic decisions on system quality. For instance, they might want to evaluate the effect of different signal-to-noise ratio characteristics on the quality of a processed audio signal. To do this, they generate real-world data for the input audio signal, apply that data to the algorithm using simulation, and save the resulting audio data.

These data can then be analyzed heuristically by 'playing' the generated audio data and quantitatively by view the signal waveforms for the generated audio. This involves applying streams of audio data (waveforms) and generating results in near real-time.

Protocol Conformance - 'Protocol' here refers to the communication protocols through which the system interacts with its environments.

In wireless communications, this includes GSM or IS-95 using lower level standards such as CDMA or TDMA. In telecommunications, this includes standards like ATM. A system is expected to accept and transmit data using these standard protocols within predefined performance windows. Today's systems are often faced with supporting multiple standards and products must adapt to geographically distinct protocol standards.

Products often must pass regulatory type approval (e.g., FCC or ETSI type approval) before being allowed into the market. If a product does not conform to a set of well-defined protocols it will not pass type approval. This can mean significant lost opportunity and revenue for the product. 


\begin{tabular}{lll} 
DESIGN LEVEL & TASKS & REPRESENTATION/CLU \\
\hline Product Specification $\begin{array}{l}\text { General Specification } \\
\text { Product Feature Selection } \\
\text { Product Constraint Identification }\end{array}$ & $\begin{array}{l}\text { Documents, Mock-ups, } \\
\text { Prior System Versions }\end{array}$
\end{tabular}

System Level Algorithm Design \& Selection FSMs and Control Languages Protocol Conformance Testing Dataflow Process Networks Concurrency analysis \& designImperative/Behavioral

Processes

$\begin{array}{lll}\text { Mapping to Target } & \text { HW/SW partitioning } & \text { Characterized Perform. Models } \\ \text { Architecture } & \text { IP Evaluation \& Selection } & \text { Communication Models } \\ & \text { System Performance Analysis } & \end{array}$

Architectural Level IP Evaluation \& Selection Cycle-Accurate C/C++ Performance Analysis HDL Models Block Compat. and Comms Boundary Characteristics (e.g. $\mu$ Architecture analysis \& design timing)

Implementation Level Gate-Level Impl.

RTL-Level verification

Area/Speed/ Power Trade-offs Selected IP (VSI components)

Timing constraint satisfaction Timing Graphs (TLF, SDF, ...)

Test Logic Insertion

Early Floorplanning (esp. for DSM)
$\mathrm{C} / \mathrm{C}++$ Software Threads

Synthesizable HDL

Detailed ASIC Sign-off verification Boolean Equations

Implementation Area/Timing/power estimation Gate-Level Models (HDLs)

Detailed Floorplanning Timing Models (TLF, SDF, $D C L)$ Schematics, EDIF etc. Footprint-level placement models, Routing estimation models, Area/Timing/Power constraints, formulae etc.)

Figure 1 - Design task clusters and their relationship to distinct representations (levels in italics not covered in this version of the paper) 
Environment Modeling - One of the most difficult tasks in system-level modeling is to model the environment in which a system must operate. As stated above, the environments for modern day electronic systems are complex and include multiple types of data (audio, video, etc.) being distributed in multiple formats (MPEG, JPEG, etc.) over multiple communication protocols (ATM, CDMA, etc.). In addition, systems are faced with highly volatile contexts in which they must operate. For instance, mobile systems must be able to maintain and optimize signal transmissions while the system is moving between different cells in the overall wireless network. To effectively evaluate the capabilities and correct functionality of a mobile handset design, the design team needs to provide real-world audio data in the right formats to the system and provide real-world interaction with the transceiver base station including the effects of moving through the cell network. It is also useful, to analyze the results of the system processing both in detailed representations and in more userfocused representations. For instance, design teams would like to analyze audio quality both through hearing the results (user-focused) and utilizing audio waveform analysis tools. This allows the team to cross-correlate the actual impact on audio quality for given algorithms and algorithmic decisions.

\subsection{Design Representation}

System functionality (including algorithms, protocols and environments) is generally described as a collection of distinct communicating processes or process networks. These processes are viewed as executing concurrently with communication between them managed through some model of communication. System functionality is often separated into the algorithmic processing functions and control functions. These two aspects of the system have different needs in terms of their communication model.

\section{Data Flow Descriptions}

Algorithmic processing is usually defined as a set of operations operating on a stream of input data and producing a stream of output data. The communication model most commonly used here is data flow semantics; i.e., a process (data flow actor) fires when new data (tokens) is available at each of its inputs. There is no notion of time in a data

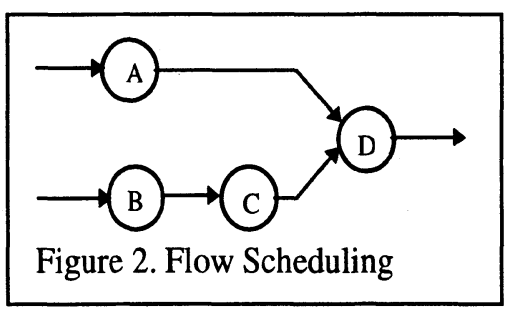
flow simulation; the goal is to transform a stream of input data into a stream of output data.

A key aspect of efficient data flow simulation is the scheduling of nodes in the process network. Processes execute data as their input tokens become available. The order in which processes must execute to ensure that their inputs are available is often 
derivable from the connectivity of the process network. For instance, consider the Figure 2. It is clear that both process $A$ and process $C$ need to execute before $D$ will be ready to execute. In addition, process $\mathrm{B}$ must execute before process $\mathrm{C}$. A schedule of $\mathrm{A}->\mathrm{B}->\mathrm{C}->\mathrm{D}$ will ensure that inputs are available when processes are execute. Static scheduling refers to schedules which can be determined before execution and dynamic scheduling is used when an input depends on the execution of the process network (usually when feedback exists). Static and dynamic data flow scheduling can be used for optimization of the simulation of data flow process networks.

\section{Control Flow Descriptions}

Control aspects at the system level are used to model the way the system responds to some external stimulus or event. Here again we use process networks. However, the behavior of these networks is quite different than that of data flow graphs. Typically, the action a process takes when it is fired is often dependent on the current state of the process. In addition, processes in control flow networks have more varied firing mechanisms; they may fire when an event (token) is detected on any one of their inputs or on some conditional combination of tokens on their inputs (including waiting for tokens on all their inputs as in data flow networks). Often, the actual process can be viewed as a finite state machine which reacts to input events by emitting new control actions for the system.

\section{Communication Modeling}

If the system description contains multiple process networks, the communication between them must be modeled. The communication path between two process networks is often represented by a queue of length 1 . This essentially provides a buffering of data which is moved from one process network to the other. Queues of different lengths can be tried to understand the communication requirements within the system. For instance, a data flow network might operate on four data inputs but the control network might be designed to produce these inputs one at a time. In this case, a queue of length 4 is required to enable the proper communication between them.

Often the communication between two networks is dependent on the state of the system or of the communication channel itself. Consider, for instance, a communication channel which might be interrupted during the execution of a data transfer. In this case, the communication channel itself can be modeled as a control process network. 


\section{IMPLEMENTATION MAPPING: PARTITIONING, ESTIMATION AND ANALYSIS}

Once a system has been defined it must be mapped onto a specific implementation or target architecture. A target architecture, at this level, is viewed as a set of processing, communication and memory resources. The goal of the mapping level is to determine the primary resources in the target architecture and analyze both the performance of the system running on the architecture and the utilization of the various resources. The mapping task consists of:

- Mapping system functional operations onto software or hardware processors or controllers;

- Mapping shared data onto the memory architecture;

- Mapping interprocess communication onto software and/or hardware communication architectures;

- Mapping system inputs and outputs into a target I/O architecture

We refer to the components of the target architecture as resources. The goal of the mapping phase is to determine the specific resources required to implement the given system . There are two ways to specify these resources: a) selecting the resources during the mapping process and deriving the target architecture from the resultant set or b) defining a proposed target architecture then mapping the system onto that target architecture. We strongly recommend the latter approach since the design team often has at least the primary components of the target architecture (processors, buses, etc.) in mind during the product design. In addition, a given electronic product design is often based on an existing implementation whether from the current design team or targeting a specific chip or chip set from an external source.

\subsection{Major Design Issues}

The mapping phase identifies the 'essential' architecture for the system. By essential, we mean that the primary architectural components (processing, communication and memory resources) but not, necessarily, all the components of the implementation architecture (e.g., peripherals, timers, etc.). The primary goal at this level is to assess the feasibility of the target architecture for implementing the system within the given constraints. We refer to this analysis as 'performance analysis' and take a broad view of the term performance. In addition, we must verify that the system is still functionally correct on the target architecture.

Hardware/Software Partitioning - One of the most critical issues in mapping a system onto a set of resources is determining whether given system functionality 
should be implemented in software (including custom or externally procured routines) or hardware (including custom implementation or externally procured cores). This issue is becoming increasingly common as system-on-chip designs almost always include programmable processors and/or controllers on-chip. In this case, the partitioning decision is driven by performance issues; software is preferred unless a hardware implementation is required to meet the performance goals of the system.

This is often the case in critical signal processing functionality which has realtime or near real-time requirements. Obviously, the performance of software implementations is highly dependent on the speed of the processor on which it is running and the compiler used to compile the software onto that processor. In addition, unless the processor is dedicated to that specific function, the scheduling and arbitration of the multiple threads whether through a custom or commercial RTOS needs to be taken into account when evaluating the performance. Also, if the software needs to read or write data, the memory hierarchy will impose additional performance variables. When looking at moving performance critical functionality onto special-purpose hardware, designers need to obtain fairly accurate estimates of the potential performance of that hardware. In addition, they need to account for data transfer to and from the special-purpose hardware block and additional control, synchronization and handshaking hardware requirements.

Resource (IP) Selection - The evaluation and selection of specific hardware and software intellectual property (IP) is critical to the overall cost and capability of the system implementation. With coming standardization to allow mix-and-match IP blocks which can be procured from multiple sources but integrated onto a single silicon substrate, the design team is left with a number of IP options. The selection of programmable IP such as microcontrollers, microprocessors and DSP processors requires projecting the performance implications on the system under design. This requires understanding the underlying programming model of the processor as well as the performance implications of how it might be integrated into the overall system (on-chip or external memory, bus interfacing, etc.). Ideally, the system software could be run on the target processor but, often, the system software is not yet developed. In addition, it is difficult to extract accurate timing information which is representative of the embedded processor running with other on-chip components.

Selection of special-purpose, non-programmable hardware blocks is more straightforward. Here the major issues are getting performance characterization data for the block. In addition, system functionality needs to be mapped onto the hardware block but the functional process in the system may not be partitioned in a way which makes this straightforward. In this case, it is important to have a functional model of the IP functionality which can be directly used at the system design level which is then mapped onto the special-purpose IP. 
Communication Mechanisms - Perhaps the most difficult aspect of mapping a system onto a target architecture is defining and analyzing the communication mechanisms through which the system's functional processes communicate. Processes mapped to software can communicate to each other through traditional memory mapping done by the software compiler. However, when software processes need to communicate to specific hardware blocks or hardware blocks need to communicate with each other, specific communication mechanisms need to be defined. Typical approaches are to implement memory mapped $\mathrm{I} / \mathrm{O}$, data bus communication, or custom interface logic.

Performance Analysis - The most important verification goal of the mapping level is the analysis of the performance of the system running on the target architecture. 'Performance' is meant in the broad sense,: namely that the system meets the overall goals and constraints of the product definition. This often includes other physical characteristics like power utilization. Performance analysis is really an assessment of the quality of the target architecture onto which the system has been mapped. During performance analysis, the design team needs to answer questions about:

- The input to output latency of the system running on the target architecture and the components which are in the critical path;

- The utilization of specific resources in the target architecture;

- The average and peak power utilization during the operation of the system;

- The impact of resource contention and bus arbitration on the overall performance of the system.

In order to perform these analyses, the design team needs to capture the constraints for the system, map the system onto the architecture and examine the estimated performance characteristics of the selected resources. They then need to select specific scenarios which will highlight information which leads to answering these questions. Scenario test sets need to be developed which will cause a) maximum data saturation of the system, b) typical data application to the system, c) maximum utilization of communication mechanisms, etc. The design teams then simulate each of these scenarios, using the results to create visual, computational, or comparative views to help answer the questions outlined above.

\subsection{Design Representation}

\section{Characterized Resources}

In order to enable performance analysis, the simulation environment needs to understand specific characteristics of the selected resources. For non-programmable processing resources and memories this information is fairly straightforward: the required characteristics include the number of cycles required to execute the resource, the static and dynamic power utilization of the resource, and the area utilization. 
These can initially be entered as 'budgets' for the resource and then incrementally updated as more information becomes available about the actual implementation of the resource.

For programmable processing resources (microprocessors, microcontrollers, DSP processors, etc.) much more information needs to be provided. This is because the performance analysis will be an estimate of the cost of running various software processes on the programmable resource. The performance of software threads running on a processor is a function not only of the processor speed but of the compiler/assembler/loader quality, operating system, memory capabilities and I/O mechanisms. These characteristics can be used to embed instrumentation into executable software which can be used in performance analysis. This is the approach taken in, for instance, the Polis (Lavagno, Sangiovanni-Vincentelli, Hsieh, 1996) project.

\section{Communication Modeling}

Communication resources have inherent performance characteristics as well. Communication between system processes can be implemented in a number of ways. If two processes communicate directly this can be handled by either shared data between software threads in the target software language or custom communication hardware (including direct wiring) between hardware blocks. Otherwise, the communication needs to be mapped onto a communication architecture which might include buses, memory-mapped $\mathrm{I} / \mathrm{O}$, and data type and/or width conversions. The performance of a communication channel is, therefore, really a function of a set of resources (e.g., data conversion block + bus interface block + bus + memory) and their interactions.

\section{ARCHITECTURAL MODELING: PERFORMANCE AND COMMUNICATION}

Systems are implemented using a combination of integrated hardware (processors, memories, special-purpose blocks and peripherals) and software components (custom software threads, predefined software libraries, operating systems and utilities) and communication mechanisms (buses, memory mapping, drivers, etc.). We refer to this as the 'target architecture' of the system. The architectural level is used to refine an initial architecture definition into an implementable architecture. This includes selecting and configuring memories, defining the detailed interconnection of architecture resources, adding in support blocks such as peripherals, defining the $\mathrm{I} / \mathrm{O}$ 
architecture for communicating with the external world, and identifying clocking requirements for synchronizing the execution of the multiple architecture resources.

\subsection{Major Design Issues}

Interblock Communication - One of the key issues at the architectural level is defining the actual communication channels in the architecture. This includes defining the connection of blocks to the communication infrastructure. Increasingly, hardware communication within the overall system will be performed on well-defined buses including on-chip buses. This allows a hardware block to be developed in some isolation from the other blocks with which it needs to communicate. On the other hand, blocks may need to be developed which can be integrated onto multiple bus standards or other communication techniques (e.g., dual-port RAMs). The design team needs to define the data communication mechanisms between blocks. This can be as simple as transfer a single data word between two blocks or as complex as a burst transmission of large data streams using communication packets. The verification challenge is to evaluate that data transmission is correct and that the timing and synchronization of the transmissions is reliable, especially when the communication is done over arbitrated communication channels. One of the most difficult interblock communication mechanisms to verify is the communication between software and hardware within the system. This communication often involves detailed understanding of status registers, memory utilization and memory maps, and the interrupt and synchronization characteristics of hardware blocks in the system. It is this communication that is forcing the issue of hardware/software coexecution at the architectural level.

Clocking and Synchronization - The various hardware resources included in the target architecture have particular and, often, distinct clocking and synchronization requirements. Custom hardware processing blocks are primarily implemented as a combination finite-state machine and dataflow path. The FSM is used to control the movement of data through the data path and it is assumed that it will be clocked externally at, at least, a specified clock rate. Most (synchronous) blocks can be executed using a slower clock rate than the assumed clock rate but not with a faster clock. Most complex architectures require multiple clocks throughout the architecture running at different (potentially unrelated) rates. The design team therefore needs to establish a clocking strategy for the overall design and define and connect these clocks into the architecture resources. In addition, many communication schemes between blocks (and between blocks and buses) are based on specific handshaking protocols and signals. The design team needs to create a communication infrastructure which ensures that these handshaking signals are generated correctly and do not create problematic issues in operation, like deadlock. 
Target Architecture Completion - In order to complete the implementation of a target architecture, the design team needs to add in all the peripherals, I/O connections, clock generators, timers, etc. In addition, the final memory sizes need to be determined and instantiated as part of the architecture.

Software Design - Embedded systems have unique constraints which differ from general software development. Among these are potential stack limitations, limited memory, programmable hardware ports, interrupt generation and handling and system initialization and configuration requirements. These characteristics require running the software with the hardware architecture to ensure that these issues are being handled correctly.

Custom Block Behavior and Interface - In any target architecture, the design team might choose to implement a custom block in-house or work with another team to implement the custom block for them. This requires the them to come up with an implementable description of the block. For hardware blocks, this generally means creating a behavioral- or RTL-level HDL description of the block, generating a set of constraints for the block (derived from the constraint budget used for the block during performance analysis), and creating a set of tests which can be used to verify that the block is working. These descriptions are generally passed into block implementation design environments consisting of high level floorplanning, behavioral or logic-level synthesis, datapath generators, timing analysis and simulation tools.

\subsection{Design Representation}

At this level, it is important to be able to identify and use specific physical characteristics of the components in the target architecture including the primary communication boundaries (hardware I/Os and software APIs), the cycle behavior of each hardware component, and communication-accurate behavior of hardware and software components.

\section{Hardware Component Modeling}

Hardware components are best represented by 'cycle-accurate' models. 'Cycleaccurate' in this context means cycle-accurate at the boundary of the hardware component. At this level, the designer will be interested in designing and verifying the clocking, control and handshaking mechanisms between the different blocks in the target architecture. The internal details of the component itself are not required. However, detailed models may be used here if those are the only models available. There are three basic approaches to modeling hardware components at this level: bus-functional models, cycle-accurate functional models and detailed implementation models. 
Bus functional models are used to generate typical bus transactions from a hardware component so that other devices on the bus can be debugged. Typically, these models are not functional in that they do not actually perform their intended operation but rather respond to and produce correct bus communication. For instance, a processor bus functional model when presented with an interrupt request from the bus might delay some number of cycles and then generate an interrupt acknowledge onto the bus. This does not mean that it is actually executing a software thread which is then interrupted.

Cycle-accurate, functional models perform their intended function synchronized by one or more clocks and/or other synchronization inputs into the model. These models are characterized by clocked buffers which store the inputs and outputs of the model.

A detailed implementation model will be cycle- and or full-timing accurate not only at the boundary of the block but also in the block internals. This is not required for the verification tasks at this level, only for verifying the actual block. However, it is often the case that these are the only available models for the design team.

\section{Software Components}

Software components are usually represented in their production code form whether this is $\mathrm{C}$, assembler or some other language. In order to run the software with the hardware architecture a number of techniques can be employed including emulation, hardware modelers, processor models and instrumented software execution.

Typically, hardware emulation is used to emulate the behavior of custom blocks being defined in the system. This hardware emulation is connected to a board holding the processor and memory being used and on which the software can be executed. This allows the design team to run the software on the processor chip and emulate the communication and behavior of the custom blocks in the system. This is a cumbersome process but worked well for ASIC-on-board development. However, as the custom blocks, processors, and memories move onto the same silicon, the communication and timing characteristics become extremely difficult to correlate between the emulation system and the resulting implementation. We expect this solution to fall short of the needs for block-based design.

Hardware modelers are similar except the custom hardware (typically described in HDL code) is executed on a software simulator communicating with an actual processor.

There has been an increasing trend toward finding ways of running software on a software model of a processor in conjunction with software simulations of the custom hardware. This is being accomplished by either hooking the software into an HDL simulator as an instantiated component through an API (e.g., Verilog's PLI) or 
running the software on an embedded software model of the processor which is usually a combination of an instruction set simulator coupled with a cycle-accurate wrapper to handle synchronized communication with the rest of the system.

\section{IMPLEMENTATION HANDOFF: THE NEW SIGNOFF PARADIGM}

The high-level design process and verification methodology must ensure that the functionality that has been designed is correct with respect to the scenarios chosen for verification and that the description of the design (structure, behavior, constraints and margins) will lead to successful implementation with high yield.

\subsection{Major Design Issues}

The nature of the data flowing in both directions across the interfaces between systems design and detailed implementation stages must meet much more exacting requirements than has been the case until now, since this is where sign-off is desired. This may entail extra steps in the design process. These new aspects of the design process raise issues and pose technical challenges that have not yet been completely addressed in theory or in practice.

Two related issues, which do not have a clear resolution, now assume great technical and contractual importance with regard to the flows of information across the sign-off interface.

IP-Block Interface Characterization - The detailed content of a block may be withheld by the IP-provider from the system designer for reasons such as protection of trade secrets or complexity beyond the system designers' needs and computational capacity for integration. As a result, characterizations and descriptions, that are tuned to the analyses and integration process of the system designer, are required. As is clear in the work of the Virtual Socket Interface (VSI Alliance, 1996), this is more straightforward the closer to the physical implementation one gets: there are good standards for much of the physical characterization of the behavior of a chip and associated constraints, at the gate level. However, as indicated by the work of Yalcin and Hayes (1995), there are significant problems in aggregating component-level timing characteristics so that meaningful margins survive at the block-level. We believe that it is the ability to characterize and estimate these margins with high quality that is the key element in assuring a clean, predictable and successful handover from the system-level design entity to the implementation entity.

On-Chip Block Characterization - Once the IP blocks that will be incorporated in the design have been selected and 'glue-logic' created that, itself, may approach the complexity of a modern ASIC ( $200 \mathrm{~K}$ gates / $20 \%$ of the gates on the chip), the task 
at the interface is to verify that the whole assembly meets product performance requirements, satisfies constraints and performs correctly under the selected test scenarios.

Given the margins on the timing data provided by the IP authors, system designers need to return a netlist and 'glue' description [possibly synthesizable RTL] along with constraints and the margins that they have calculated or estimated. The design must have reasonable margins acceptable for product performance and manufacturability, and be well-centered within these margins. The verification problems that need to be solved are those of interface consistency for each block and interface compatibility between blocks, which involves verifying the feasibility of their handshake protocols within a framework of timed-protocol constraints as well as the evaluation of the tightness of the timing-margins around control and data edges.

Verification at the interfaces between blocks and its separation from verification internal to the blocks leads to a divide and conquer verification strategy which shares the responsibility between author and integrator, as well as reducing the overall complexity of system-wide verification. The emerging availability of constraint verification techniques (Khordoc, K. and Cerny E., 1996) applied only to expected interface protocols should further simplify the verification problem.

\subsection{Design Representation}

\section{Hardware Components}

The Virtual Socket Interface specification (VSI Alliance, 1996) has a characterization of hardware component descriptions that covers most flows. In essence, IP comes in three major flavors. 'Soft' is represented in HDL that may include behavioral and synthesizable code. 'Firm' adds floorplanning and other data that give better guarantees for successful implementation in at least one process technology. 'Hard' is delivered as a mask-level implementation that is accompanied by full timing and electrical characterizations and that may have HDL models describing behavior at a high level but, almost certainly, not implementation details. According to IP flavor, the representation may include any of the following: HDL (behavioral, RTL, gate), synthesis scripts, floorplan for custom blocks, boundary characterization (timing diagrams, etc.), etc.

Space limitations force us to refer the reader to the Virtual Socket Interface specification for hardware, software and system configuration representations. 


\section{INTEGRATING LEVELS}

In each of these four levels, from system functionality to implementation handoff, there are specific design representations and verification techniques which are appropriate for the required methodologies and tools. When working at any level, it is most efficient to work with the design representation most appropriate for the task at hand. However, it is important to be able to move between levels, to reuse models and to define a smooth transition of the design representation as we proceed from level to level.

It is beyond the scope of this paper to go into the transformation process between each of the levels. There are a number of key sources which describe the role of synthesis including De Micheli (1994), Gajski, Dutt, Wu, Lin (1994)

\subsection{Mixing Representations}

In real-world situations, design teams need to deal with mixed representations of various components which make up a system. This occurs in two ways: a) available models only exist in representations other than the one in which the design team is working or b) design teams are incrementally refining portions of the design during a transformation process.

\subsection{Test Bench Methodologies}

A design team is faced with the necessity of verifying the design at multiple levels of abstraction. The overall verification approach for a design usually requires generating sets of tests to apply to the design at each level of abstraction. These test sets are derived from the scenarios which target the aspects of the design being verified at any given level.

In order to best leverage the multiple scenario test sets developed by the design team, an integrated test bench methodology should be employed which allows the team to reuse and correlate results between levels. This requires setting up a shared verification environment which can apply different test sets to the design under direction from the design team. This, in turn, requires that the test bench be robust enough to handle test sets targeted at different aspects of the design. In addition, it may be necessary to convert the stimulus generated by the verification environment into a form appropriate to the boundary of the aspect of the design being verified.

Test set generation is a challenging problem. It is important to distinguish two types of test generation: generation of real-world data for application to the design and generation of test sets for specific aspects of an internal implementation of a system block. 
As mentioned earlier, test scenarios are a design dependent combination of stimulus generators or test vectors, results analyzers or comparators and a test bench infrastructure. However, the stimulus generation and results analysis can be constructed using application domain specific building blocks. For instance, the generation and analysis of ATM packets can be used for a large number of ATMbased designs.

The decomposition of the verification problem is important to control the application of test scenarios to the design. It is unfeasible to run all the test scenarios on every representation of the design.

\subsection{Mixed-Level Verification Technologies}

Since IP-based design is really complex system design, it will be rare to find a design which is completely defined using a single modeling technique. For instance, system level design mixes environment modeling with discrete-event and dataflow representations of a design. Because there will be heterogeneous descriptions, cosimulation techniques will be required.

Historically, cosimulation has been viewed as utilizing lower level designs in higher level simulations (e.g., gate level descriptions inside RTL designs, analog models inside digital designs, etc.) or mixing multiple simulators at the same level.

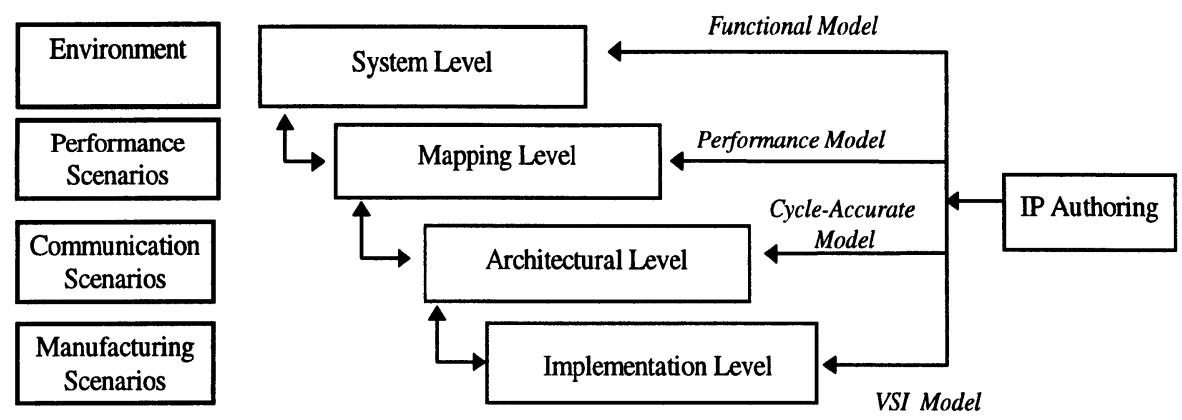

Figure 3. Integrating Levels

One aspect which is often ignored is the reuse of higher level models at a lower level of abstraction. This can happen for two reasons: the need to reuse a higher level environment model to verify a lower level representation of a design or to include high level descriptions in a simulation for parts which have are not refined to the current level. Sometimes only higher level models exist for a component which is needed for a simulation of the overall system. 


\section{CONSEQUENCES FOR HDLS}

The discussion in this paper has significant implications for HDLs and their role in the overall design and verification process. Each design phase or level requires multiple models and robust verification strategies. The analysis and verification methodologies used at each level are clustered around a collection of design representations most natural for that level. Furthermore, each level addresses issues and concerns that are distinct and largely disjoint. Thus in representing designs, a layered modeling and verification approach seems most appropriate.

In general, effective design and verification methodologies for system-on-a-chip design are not HDL-based; HDLs can be used to represent certain aspects of a design within an overall flow but they are not the primary, or even predominant, component of an overall design representation.

The designers of VHDL were explicit in their intention to create a language that reflected that which could be implemented as hardware: they were successful. VERILOG is no different in this regard. With the passage of time, requirements have evolved and while we do not believe that an attempt to extend HDLs arbitrarily up into systems space would be fruitful, there are some shortcomings that could be addressed and some existing properties of HDLs that have become redundant and whose elimination could lead to simplification.

HDLs are not general purpose description languages but are mainly focused on discrete-event simulation. Subsets have been extracted to support logic synthesis. As a result, there is no well-defined support for the application of analyses by means other than simulation.

There is very weak support for the specification and management of requirements and constraints. In this respect the emerging work on VSPEC (Baraona et al., 1995) and RAPIDE (Luckham et al., 1995) is of interest.

While HDLs support the notion of a logic signal, they make little provision for the description of connections at higher levels of abstraction. As a result of this and similarly restrictive semantics for other aspects of designs, the description of a system has to be close to an implementation in order to make use of current HDLs.

The description of sequencing and timing is embedded in the operational model and is implicit. This limits the usefulness of HDLs for system-level description especially for asynchronous behavior and reactive systems.

These observations indicate some of the significant obstacles for HDL use at the System and Mapping levels, and an unproductive misfit at the architectural and implementation levels where cycle-accurate simulation and static timing analysis are being adopted as verification methods of choice. 
Even if no attempt is made to extend the reach of HDLs into system and architectural domains, the smooth integration of HDL-based sub-flows with higher level flows requires that these kinds of issues be addressed. The alternative is a plethora of piecemeal additional representations that fit badly together at the boundaries of design model clusters, requiring expensive extra conversion, reconciliation and re-modeling.

System Level

At the system level, the analysis of designs is in terms of data flow and control flow. As we saw, the most effective design representations at this level are data flow process networks and networks of reactive control processes. By definition, data flow models are stream-based; i.e., data flow networks are applied to streams of data. A dataflow actor fires when all of its inputs become available. While syntactically, HDLs can be used to describe such process networks, the underlying semantics of the inter-process communication model of HDLs require explicit modeling of the communication between processes.

In addition, one of the key issues in system design is data representation. Since HDLs have either predefined data types (Verilog) or strong typing (VHDL) with no polymorphism, it is difficult to refine the data types of communication channels between processes. For instance, in refining a VHDL entity for a data flow process (e.g. a DSP application) from floating point types to a fixed point representation, the entity must be rewritten, recompiled and a new architecture selected for the functionality. This cumbersome process is error-prone and workarounds lead to very inefficient design representations.

\section{Mapping Level}

At the mapping level there are three distinct modeling challenges when using HDLs. The primary change in description between the system-level model and the mapping level model is the need to map the system processes onto specific hardware resources and analyze the resulting performance. The only way to do this in HDLs is to embed the processes for a given resource into an HDL description of the resource itself. This causes a dramatic change in the system hierarchy and significant modeling effort. To change the allocation requires reworking the structural hierarchy of the design in major ways. This does not realistically permit effective analysis of different mappings of the system onto the architecture.

Another important challenge, is that the focus at this level is on the impact of selecting resources. This only requires injecting critical performance characteristics of the resources into the performance analysis, without requiring models of the resource implementations.

Finally, modeling software using HDLs is counter-intuitive. Software interfaces are usually defined as subprograms with parameters (APIs), or objects with publicly 
available methods. Interface definitions in HDLs are foreign and cumbersome for software-oriented descriptions.

\section{Architectural Level}

While HDLs have some distinct advantages at the architectural level, there are some major modeling issues. In their favor, HDLs are well-suited for defining robust generation of clock and synchronization signals and connecting together blocks through well-defined interfaces. In addition, VHDL configurations are useful for making modifications to the overall structural description of the architecture. However, the synchronization and scheduling semantics are fixed to basic models which do not match the actual hardware activity well. For instance, there is no distinction of clock or synchronization signals from general signals. Also, in order to model any communication scheme other than direct wiring, the user must add new and different structural elements into the design even when no such structure will exist in the actual architecture.

Perhaps most troublesome at the architectural level is the event-driven orientation of HDLs. This shows up most dramatically in the timing and scheduling semantics of various HDLs. As we stated above, designs at the architectural level should be represented by cycle-level semantics and cycle-level models. This means that data transfer and process synchronization should only be tied to clocking and/or handshaking signals. However, HDLs have intimately bound the assignment of data into communication channels between blocks with the time it takes to propagate that data through those communication channels. As has been shown in Rowson and Sangiovanni-Vincetelli (1997), there is an increasing need to separate behavior and communication in an overall system design.

\section{Implementation Level}

At this level there are two areas of concern, particularly for block-based design flows: what the IP authors provide as block characterizations; and how the system integrators gain assurance that the design representation that they sign-off for implementation, will in fact lead to a high yield, correct product.

System integrators and consumers of IP need a representation of timing and function and testability as seen at the external interfaces of blocks that is adequate to ensure that connecting blocks are compatible with respect to their handshake protocols, that the timing margins have enough slack to ensure acceptable manufacturing yield and that the ensemble will be testable.

The reader is referred to the VSI Specification (VSI Alliance, 1996) which makes it abundantly clear, that there is a plethora of additional data - physical, timing, scripts for synthesis, etc., according to the kind of IP involved - that must be provided to the system integrator for a sign-off to be robust. 


\section{ACKNOWLEDGEMENTS}

A number of individuals and organizations have shaped the content of this paper including Jim Rowson, Patrick Scaglia, Oz Levia, and Alberto SangiovanniVincentelli.

\section{REFERENCES}

Baraona, P., Penix, J. and Alexander, P. (1995) VSPEC: A Declarative Requirements Specification Language for VHDL. In Current Issues in Electronic Modeling, Kluwer Academic Publishers, Vol. 3, 51-75.

Boriello, G., (1988) A new interface specification methodology and its application to transducer synthesis. Ph.D. thesis, University of California-Berkeley.

Gajski, D.D., N.D. Dutt, C.H. Wu, and Y.L. Lin (1991) High Level Synthesis: Introduction to Chip and System Design. Kluwer Academic Publishers.

Khordoc, K. and Cerny, E. (1996, To Appear) Semantics and Verification of Action Diagrams with Linear Timing Constraints. ACM Transactions on Design Automation of Electronic Systems.

Lavagno, L., A. Sangiovanni-Vincentelli, H. Hsieh, (1996) Embedded System CoDesign. In Hardware/Software Co-Design, pp. 213-242, Kluwer Academic Publishers.

Luckham, D.C., Kenney, J.J., et al. (1995) Specification and Analysis of System Architecture Using Rapide. IEEE Transactions on Software Engineering, 21(4):336-355.

Rowson, J. and A. Sangiovanni-Vincentelli (1997). Interface-Based Design. To be published.

Shi, C., Shenghua, H., Lien, Y.E. (1996) Using the C Language to Reduce the Design Cycle of an MPEG-2 Video IC: A Case Study. $2^{\text {nd }}$ International Conference on ASICs, Shanghai.

VSI Alliance (1996) Virtual Socket(TM) Interface proposal. Revision 0.9.0.

Yalcin, H. and Hayes, J.P.(1995) Hierarchical timing analysis using conditional delays. Proc. ICCAD, 1995.

\section{BIOGRAPHIES}

Cary Ussery is Group Director in the ALTA group at Cadence. He earned a B.A. in mathematics and a B.A. in Music at Bard College, N.Y. in 1984. He is co-author of "VHDL: Hardware Description and Design", Kluwer, 1987.

Simon Curry is architect in the Design Flow Engineering group at Cadence. He earned his Ph.D. in Electrical Engineering Science at the University of Essex in 1975. 